\title{
OPTIMAL LASER WAVELENGTH FOR RESONANT TRANSMISSION THROUGH THE COULOMB BARRIER
}

\author{
ROBERT W. BASS \\ Innoventech Inc., P.O. Box 1238, Pahrump, NV 89041-1238 \\ E-mail:donquixote@innoventek.com
}

\begin{abstract}
The 1993 point-particle, 1-D semi-classical quantum-mechanical theory of resonant transparency of the Coulomb barrier [1], though admittedly crude, has some plausibility as to relevance and adequacy because it predicts quantitatively different results for lattices of protons versus deuterons embedded in host lattices of palladium versus nickel. Specifically it predicts that cold fusion is likely with $\mathrm{Ni}$ cathodes and electrolysis with either ordinary or heavy water, whereas it is likely only for heavy-water electrolysis with Pd cathodes. The question of whether or not this 1993 theory has any predictive power regarding the 2003 Letts-Cravens effect is considered and answered as: "partially." That is, the "optimal" range of wavelengths includes that discovered by Letts and Cravens, but because of the possibility of multi-photon reactions it does not uniquely single out their $680 \mathrm{~nm}$ as best, which would require a more detailed model (including e.g. cathode temperature) than provided by the simplified 1993 theory.
\end{abstract}

\section{Introduction}

The 1993 theory [1] of resonant transparency of Coulomb barriers in a periodic lattice will be reviewed and improved quantitatively and then analyzed regarding its consistency with, and relevance as a crude prediction of, the celebrated 2003 Letts-Cravens Effect.

\section{One-dimenional model}

Briefly, the host lattice is ignored and the embedded-particle lattice is modeled as an electrically neutral linear 1-dimensional lattice (see Figure 1) consisting of alternating bound ions and "averaged" bound electrons, except on the central interval of interest which is of width $2 L$, where $L$ is the arbitrarily-specified lattice spacing (assumed derived from the host lattice). ${ }^{a}$ On this interval, 3 electron charges are modeled as a smeared-out distribution while the adjacent ions to right and left are regarded as bound but the approximately central ion is regarded as both excited and off-center; then by summation of obvious infinite series an electrostatic potential

a A summary of the theory in question was presented recently at the MIT Cold Fusion Colloquium and is available upon request from the author as a PowePoint slideshow. 


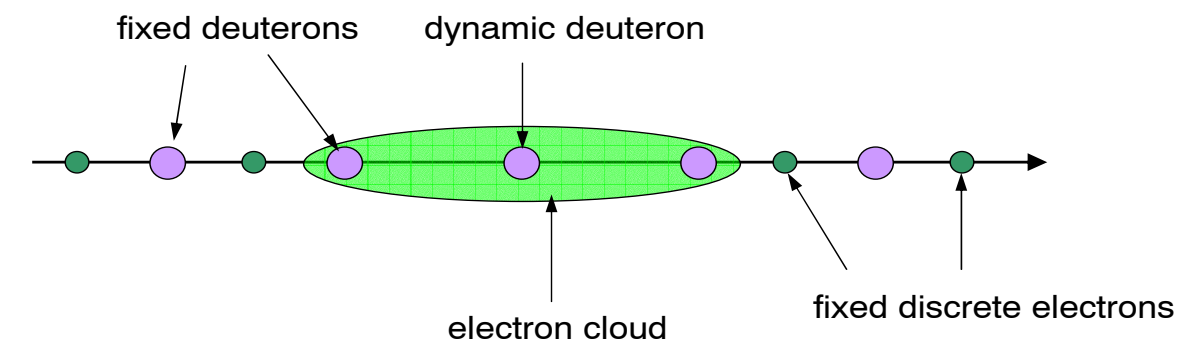

Figure 1. Schematic of the one-dimensional model under discussion. The model focuses on a quantum physics of a single deuteron (labeled dynamic deuteron), with neighboring deuterons assumed to be fixed. The electronic contribution in the vicinity of the dynamic deuteron is taken into account as an equivalent potential. Other electrons are taken into account using a fixed charge model as indicated.

$$
V=V(r)=V(r+2 L)=V(r-2 L)
$$

is defined for $[-L, L]$ and extended by definition to the entire lattice by the preceding requirement of periodicity; this is a Coulomb/Madelung/Fermi-Thomas-Mott potential as used by Mott in solid-state physics. The validity of this potential is tested by calculation of the empirical Schwinger Ratio [1], which predicts a result that is $99.7 \%$ of the measured result in Pd. $\mathrm{D}_{1.0}$.

\section{Discussion}

Using a generalization of the Gamow theory presented in the classic book of Bohm, it is proved that the analytical condition for the excited ion to be bound (when it is considered that there are Coulomb barriers to the left and to the right only) is identical to that for the adjacent barriers to be resonantly transparent when there is a periodic array of such barriers, as in the present model. In the 1993 paper [1] the first few hundred energy levels corresponding to resonant transparency were computed, both by hand and by use of Mathcad, and a quartic fit to the 20 'best computed' values was made. Then 10 cases of ZPF-induced line-breadth were computed and a quartic fit to this function (of the index $n$ representing the integral multiple of the lowest energy level) was also made. As shown in the Appendix, greater numerical accuracy has now been obtained using the MATLAB program and data given below, with the result that the ZPF-induced line-broadening is greater than $50 \%$ between $n=99$ and $n=336$, which corresponds to the energyinterval $17.6<E<60.8 \mathrm{eV}$, with line-breadth greatest at $n=190$ where $E=30.9$. This range of energy-levels corresponding to a continuum spectrum rather than a discrete line-spectrum seems to be related to the ion band-state theory of Chubb and Chubb [3,4] and is of interest because only a line-spectrum was expected and the continuum-spectrum in the center of it had neither been expected nor foreseen by me when I started with the model used by Julian Schwinger and attempted to 
improve it with concepts gleaned from many others, including Leaf Turner, Robert D. Bush, Willis Lamb and Robert Parmenter, etc.

Considering the fact that these calculations were based upon numerical differentiation of a quartic fit, no great precision is claimed, so I will round off the figures to be discussed. However, my 1993 result is that if one considers the excitation of a deuteron by a single high-energy photon, then the energy range of $[18,61] \mathrm{eV}$ corresponds to a wavelength range of about $[20,70] \mathrm{nm}$ with the alleged "optimal" energy of about $31 \mathrm{eV}$ corresponding to $40 \mathrm{~nm}$.

In my 1993 paper I had written (p. 38-1) "Alternative versions of the experiment would replace the deuteron beam by a high-intensity flux of coherent monochromatic ultraviolet rays providing photons of energy $\mathrm{E}_{\text {crit }} . .$, , and on page 38-4 elaborated on the possible use of "UV lasers" and "continuous tuning of the wavelength" of "chemical lasers (such as are based upon liquid organic dyes)."

Unfortunately I was not then familiar with fact that heavy water is not transparent to UV lasers and that it is far more convenient experimentally to use optical wavelengths (particularly red light). I wish to thank Michael Ibison (assuming that I understood his concise verbal remarks correctly) for pointing out that the excited deuteron can be hit by more than one photon, and can have its energy level raised over and over until it finally reaches a level at which resonant transmission occurs. Therefore I might have postulated multi-photon reactions involving 10-to-20 photons, which would have enabled me to suggest starting with wavelengths in a visible range such as $[400,1400] \mathrm{nm}$ as more practical than UV.

\section{Connection with Letts experiment}

Letts has found (and been confirmed by Storms et al) that $680 \mathrm{~nm}$ seems to be optimal, which corresponds to photons of energy $1.81 \mathrm{eV}$. If one considers a cascading effect involving simultaneous (or even sequential) impingement upon the excited deuteron by from 10 to 20 photons, this would produce an excited deuteron in the energy range of $[18,36] \mathrm{eV}$ which lies within my desideratum of $[18,61] \mathrm{eV}$ and includes my previously claimed "optimal" energy level of $31 \mathrm{eV}$. In fact $17 \times 1.81$ $\mathrm{eV}=30.8 \mathrm{eV}$, in good agreement with my 1993 value [1] of $30.9 \mathrm{eV}$.

Accordingly one may perceive in hindsight that my 1993 theory [1] is consistent with the Letts-Cravens discovery, but not sufficiently refined to have been able to predict it specifically and uniquely. Indeed, Letts and Cravens have found that their effect is quite sensitive to variations in the temperature of the cathode, which is an effect entirely omitted from my crude approximation of the ion lattice embedded in a host lattice. Only further research can hope to produce a sufficiently detailed analytical model from which a uniquely best laser-trigger wavelength might be predicted theoretically.

\section{References}

1. R. W. Bass, "Proposed nuclear physics experiment to conclusively demonstrate and explain aneutronic cold fusion, " Proceedings of the Fourth International Conference on 
1016

Cold Fusion, Dec. 8-9, 1993, Volume 4, p. 38-1; available in 4 volumes from EPRI Distribution Center, 207 Coggins Drive, P.O. Box 23205, Pleasant Hill, CA 94523; or contact Simon at askepri@epri.com.

2. R. W. Bass, "Resonant Transparency Spectrum of Deuterium Lattices in $\mathrm{PdD}_{1.0} \mathrm{CF}$ Reactors," Proc. ICCF5 (Poster Paper), Monaco (1995).

3. S. R. Chubb and T. A. Chubb, Proc. ICCF8, 385-390 (2000).

4. T. A. Chubb and S. R. Chubb, Proc. ICCF8, 391-396 (2000).

\section{Appendix}

function [En,dfEn,dEn,deltaEn,BroadRat $]=$ RTSpectrumAppx3(n);

$\% * * * * * * * * * * * * * * * * * * * * * * * * * * * * * * * * * * * * * * * * * * * * * * * * * * * * * * * * * * * * * * * * * * * * * * *$
$\%$
$\%$ Inputs:
$\% \mathrm{n}=$ integer, $0</=\mathrm{n}</=600$
$\%$ Outputs:
$\%$ En $=$ nth energy level of Resonant Transparency Spectrum,
$\%$ from quartic fit to 20 best-computed values
$\%$ dfEn $=$ Enp - En, where $\mathrm{np}=\mathrm{n}+1$
$\%$ dEn $=$ Line-Breadth (induced by ZPF), fit to 10 values
$\%$ BroadRat $=$ dEn. $/ \mathrm{dfEn}, \mathrm{using}$ preceding two quartic fits
$\%$ deltaEn $=$ quartic fit to 10 best-computed values of BroadRat
$\% \mathrm{E}(191)=31.0342 \mathrm{eV}=\mathrm{E}(190)+0.1656 \mathrm{eV}$
$\% \mathrm{E}(190)=30.8681 \mathrm{eV}$ is approx max of BroadRat in that line-breadth
$\% \mathrm{dE}(190)=0.0984$ is $59.43 \%$ of distance 0.1656 to next line
$\% \mathrm{Note}$ that dEn $>0.5$ for $99<\mathrm{n}<337$ (at UCCF5: $95<\mathrm{n}<360)$
$\%$
$\% * * * * * * * * * * * * * * * * * * * * * * * * * * * * * * * * * * * * * * * * * * * * * * * * * * * * * * * * * * * * * * * * * * * * * * *$

$\mathrm{Eo}=6.28361787789068 ; \%$ (at ICCF5:) 6.28

$\mathrm{dE} 1=0.10239735208775 ; \%$ (at ICCF5:) 0.1024 ;

$\mathrm{dE} 2=0.00008512851650 ; \%$ (at ICCF5:) 8.516e-5;

$\mathrm{dE} 3=0.00000033805923 ; \%$ (at ICCF5:) 3.38e-7;

$\mathrm{dE} 4=0.0000000020174 ; \%$ (at ICCF5:) 2.016e-10;

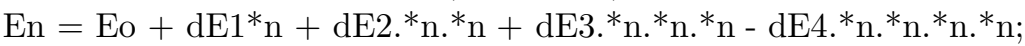

$\mathrm{np} 1=\mathrm{n}+1$

Enp1A $=$ Eo + dE1*np1 + dE2.*np1.*np1;

Enp1B = dE3.*np1. ${ }^{*} n p 1 .{ }^{*} n p 1-$ dE4.*np1.*np1.*np1.*np1;

Enp1 = Enp1A + Enp1B;

dfEn = Enp1 - En;

DEo $=0.00249297142391 ; \%$ (at ICCF5:) 0.0025;

$\mathrm{DE} 1=0.00078210671613 ; \%($ at ICCF5:) $7.821 \mathrm{e}-4$;

$\mathrm{DE} 2=0.00000170979858 ; \%$ (at ICCF5:) 1.71e-6;

DE3 $=0.00000000135819 ; \%$ (at ICCF5:) 1.36e-9;

$\mathrm{dEn}=$ DEo + DE1 ${ }^{*}$ - DE2. ${ }^{*}$ n. ${ }^{*}$ n + DE3. ${ }^{*}$ n. ${ }^{*}$. ${ }^{*}$; 
BroadRat $=$ dEn. $/$ dfEn;

dlEo $=0.02399512497696 ; \%$ (at ICCF5:) 0.0165;

$\mathrm{dlE} 1=0.00777727865775 ; \%$ (at ICCF5:) 0.00896 ;

dlE2 $=0.00003571015455 ; \%$ (at ICCF5: $4.93 \mathrm{e}-5$;

dlE3 $=0.00000006320703 ; \%$ (at ICCF5:) 1.05e-8;

$\mathrm{dlE} 4=0.00000000003942 ; \%$ (at ICCF5:) 7.7e-11;

deltaEn $=$ dlEo + dlE1*n - dlE2. ${ }^{*}$ n. ${ }^{*}$ n + dlE3. ${ }^{*}$ n. ${ }^{*}$ n. ${ }^{*}$ - dlE4. ${ }^{*}$ n. ${ }^{*}$ n. ${ }^{*}$ n. ${ }^{*}$;

$\%$ end of RTSpectrumAppx3.m 